\section{Relevancia de profilaxis antibiótica ante procedimientos dentales generadores de bacteriemias transitorias}

\author{
ENRIQUE FERNÁNDEZ ${ }^{\mathrm{a}}$, CHRISTOPHER REYES ${ }^{\mathrm{a}}$, \\ CAROLINA BENAVIDES ${ }^{\mathrm{a}}$, TAMARA IRARRÁZAVAL ${ }^{\mathrm{b}}$, PAULA PADILLA $^{\mathrm{a}}$
}

Facultad de Odontología,
Universidad San Sebastián.
Santiago, Chile.
aCirujano Dentista.
bAlumna de pregrado
Odontología. Universidad
Autónoma. Santiago, Chile.
Trabajo no recibió
financiamiento.
Los autores declaran no tener
conflicto de interés.
Recibido el 5 de febrero de 2018,
aceptado el 6 de julio de 2018.
Correspondencia a:
Dr. Enrique Fernández M.
Facultad de Odontología,
Universidad San Sebastián
Avenida Bellavista \#7. Santiago,
Chile.
enrique.fernandez@uss.cl
Material suplementario en versión
digital de la Revista Médica de
Chile en: http://scielo.conicyt.cl

Facultad de Odontología, Cirujano Dentista. Odontología. Universidad Autónoma. Santiago, Chile.

Trabajo no recibió

Los autores declaran no tener

Antimicrobial prophylaxis for transient bacteremia during dental procedures

This review examines the evidence about the relationship between dental procedures and the incidence of transient bacteremia. One of the main obstacles was to define "invasive dental procedure" as an indication for antimicrobial prophylaxis for patients with high risk of bacteremia. A search in WorldWideScience and ScienceDirect was performed and 20 articles were selected for review. Two contradictions stood out. There is no concrete evidence that a transient bacteremia arising during a dental procedure may be a risk factor for the appearance of bacterial endocarditis. There is no certainty about the effectiveness of antimicrobial prophylaxis, due to the lack of clinical trials of good quality. There is a similitude between bacteremia resulting from invasive and non-invasive dental procedures. The importance of periodontal health as a preventive measure for bacterial endocarditis among high risk patients is highlighted.

(Rev Med Chile 2018; 146: 899-906)

Key words: Antibiotic Prophylaxis; Bacteremia; Dentistry; Endocarditis, Bacterial; Viridans Streptococci.

\section{L}

a endocarditis infecciosa (EI) es una afección cardíaca poco común y presenta de 3 - 7 episodios cada 100.000 habitantes por año en Estados Unidos y Europa ${ }^{1-4}$. En Chile, la incidencia de casos es de 2-3 cada 100.000 habitantes, con variaciones en Santiago y la región de la Araucanía, con 2 y 1,8 casos cada 100.000 habitantes respectivamente, y una mortalidad de $29 \%$, superior a series extranjeras que reportan $25 \%$ 5,6. Se ha observado un incremento en la edad media de presentación y en pacientes que poseen prótesis valvular o algún dispositivo cardíaco ${ }^{4}$. También se registra un aumento en la incidencia por Staphylococcus aureus como agente causal principal, similar a lo estudiado en Santiago ${ }^{5}$ y en la quinta región ${ }^{7}$, en donde se produce un cambio de posición con el agente Streptococcus viridans, situándolo en segundo lugar ${ }^{4}$.
Las primeras analogías en relación a procedimientos quirúrgicos y EI fueron publicadas por Osler en 1885, quien notificó una posible asociación positiva entre bacteriemia y endocarditis ${ }^{6}$. Posteriormente, las investigaciones enfatizaron en investigar las bacteriemias desarrolladas bajo distintos procedimientos quirúrgicos -incluidos los dentales ${ }^{7}$ - creando y promoviendo el desarrollo de protocolos de antibioterapia para la prevención de esta enfermedad, las cuales tuvieron su primer paso con la guía AHA publicada en el año $1955^{8}$.

En la actualidad, diversas sociedades científicas de cardiología como la "American Heart Association" (AHA) y "European Society of Cardiology" (ESC), proponen esquemas de profilaxis antibiótica (PA) como medida preventiva de EI en pacientes considerados de alto riesgo cardíaco -pacientes con cualquier tipo de válvula 
protésica (incluyendo transcatéter) o en los que se haya usado algún tipo de material protésico para reparación valvular cardíaca. Pacientes con episodio previo de EI, pacientes con cardiopatía congénita (cualquier forma cianótica), pacientes con cardiopatía congénita reparada con material protésico (ya fuese quirúrgica o percutáneamente) hasta 6 meses tras el procedimiento (o de por vida si queda shunt residual o insuficiencia valvular). Pacientes con desarrollo de vicio valvular y pacientes con transplante cardíaco- sometidos a procedimientos odontológicos definidos como invasivos (manipulación del tejido gingival, acceso a región periapical o perforación de la mucosa bucal $)^{11-13}$.

Sin embargo, este paradigma no carece de polémica. El organismo británico, "The National Institute for Health and Care Excellence" (NICE) publica en el año 2008 su guía de recomendaciones (un año después de los esquemas propuestos por la AHA), declarando que los procedimientos dentales, independiente de su riesgo, no necesitan $\mathrm{PA}^{14}$. A pesar de lo anteriormente declarado, en 2016 se establecieron modificaciones, no recomendando la PA de forma "rutinaria" para procedimientos dentales invasivos, pero resaltando la importancia del criterio clínico y la educación del paciente sobre su enfermedad ${ }^{15}$.

Paralelamente en Estados Unidos de Norteamérica, "Cochrane Institute" -red colaborativa internacional de investigadores de ciencias de la salud, reconocido por su prestigioso nivel científico- también manifiesta una posición disidente respecto de la indicación de estos esquemas profilácticos. En 2013 efectuó una revisión sistemática que arrojó como resultado la inclusión de un solo estudio clínico, concluyendo por ende, falta de evidencia científica en concordancia con los efectos positivos o negativos de la PA en función de la relación de EI y procedimiento dental invasivo, postulado similar a lo expuesto por $\mathrm{NICE}^{16}$.

Queda así definido un eje o línea de razonamiento, que transcurre desde un polo, paciente susceptible -condición cardiópata de riesgo-que expuesto a una bacteriemia originada en procedimiento dental invasivo, debe recibir profilaxis antibiótica para así evitar la generación de EI. Revisaremos a continuación los fundamentos de las discrepancias de estas agencias en torno a este primer eje de análisis de índole médica, complejizando además el debate, al agregar otra variable propia de la especialidad estomatológica, como resulta ser la polémica relativa a qué procedimiento odontológico puede tipificarse como invasivo (Figura 1). El anterior análisis lo llevaremos a cabo mediante una revisión de ensayos clínicos publicados en los últimos 10 años, que fueron dando cuenta de las actualizaciones en las líneas de investigación en torno a la bacteriemia tras procedimiento dental y consiguiente al gran cambio establecido hace una década por la AHA y la NICE, ambas entidades científicas con influencia mundial en el desarrollo de guías clínicas y promotoras de estos paradigmas.

\section{Material y Método}

Se llevó a cabo una búsqueda en las bases de datos MEDLINE (criterios de búsqueda MeSH),

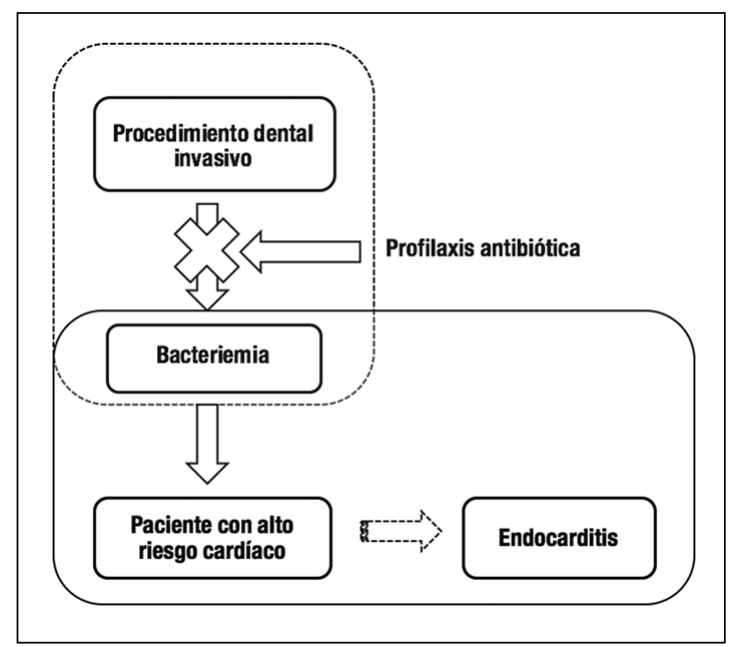

Figura 1. Representación de parámetros para indicar profilaxis antibiótica. — Eje odontológico: Define "procedimiento invasivo" a cualquier intervención que involucre: manipulación del tejido gingival, acceso a región periapical o perforación de la mucosa bucal. Esto basado en estudios que analizaban bacteriemia transitoria después de un procedimiento dental, y donde se determinaba que una intervención "invasiva" es aquella que generaba una bacteriemia mayor que en procedimientos de rutina odontológica9,10 - - - - - Eje médico: Describe a un paciente de "alto riesgo cardiaco" con las siguientes características: pacientes con cardiopatía valvular adquirida o congénita, miocardiopatía hipertrófica, prolapso mitral con insuficiencia valvular, prótesis valvular o antecedentes de El. Si un paciente con tales características, se expone a una bacteriemia por procedimiento dental invasivo, podría eventualmente generar una endocarditis bacteriana. Por lo que, indicar profilaxis antibiótica, sería la mejor opción ante la posibilidad de desarrollar $\mathrm{El}^{9,10}$. 
WorldWideScience y en ScienceDirect con las palabras claves: "Endocarditis, Bacterial" y "Dentistry", con sus símiles en español. Cabe resaltar que el criterio de inclusión para esta búsqueda bibliográfica fueron aquellos estudios clínicos randomizados, textos completos y publicados entre los años 2007 y el 2017. En la Figura 2 se detallan las características del algoritmo utilizado.

\section{Resultados}

La búsqueda arrojó un total de 1.855 artículos, filtrando 226 textos de acuerdo a los criterios de inclusión, para finalizar tras los criterios de exclusión en 20 publicaciones. En la Tabla 1 se presenta el resumen de estos estudios, los cuales corresponden a las distintas especialidades odontológicas que

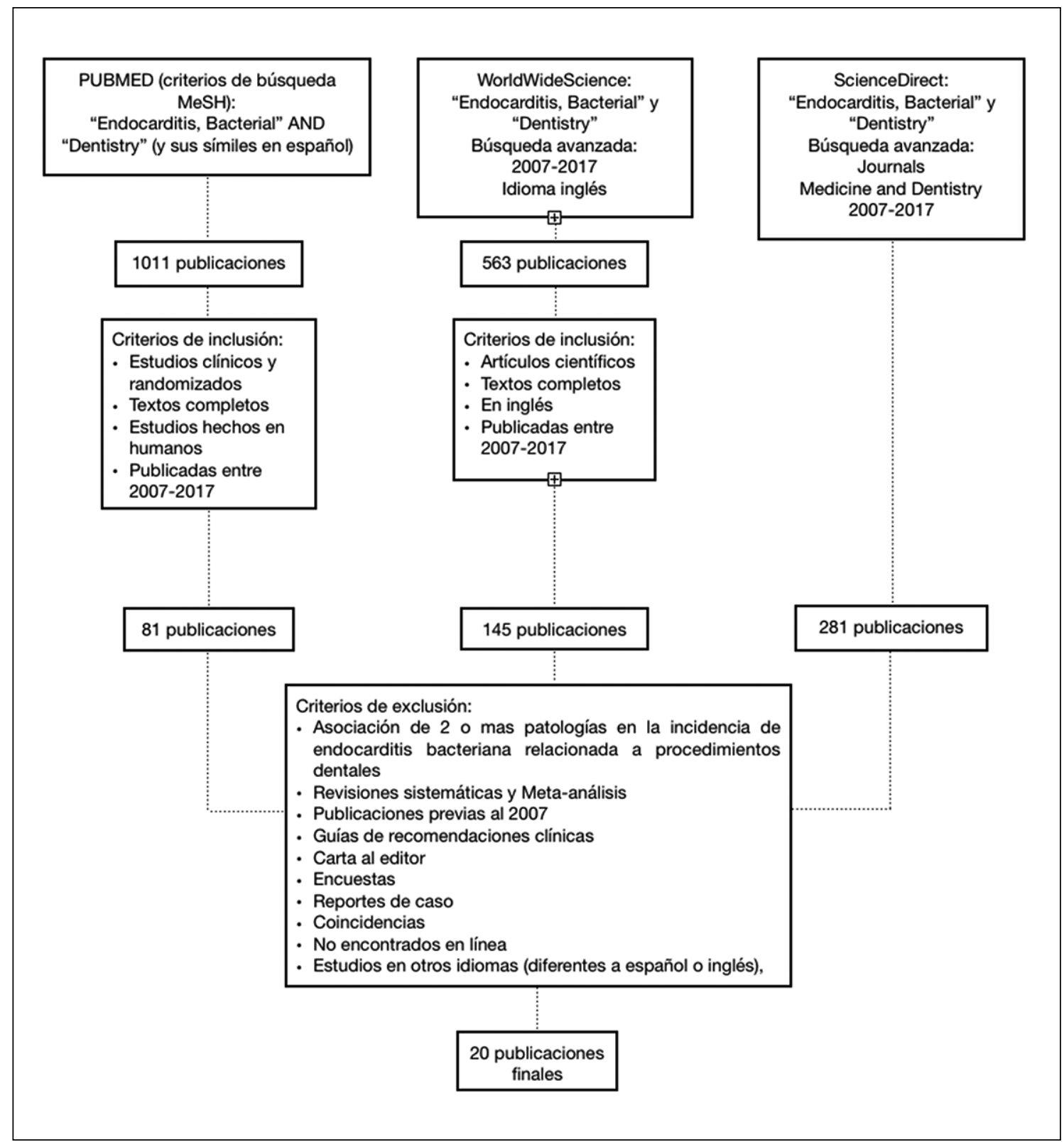

Figura 2. 
apoyan y cuestionan la indicación de PA, basados en la presencia del microorganismo Streptococcus viridans, grupo asociado históricamente a bacteriemia de origen oral ${ }^{11,12}$. En general, ésta se detecta a los pocos minutos de realizada una intervención invasiva (i.e. exodoncia) $)^{17-19,21,22,28,32,34,36}$; destacando que la presencia de bacteriemia en los cultivos aún persistía en un porcentaje considerable y similar a otros procedimientos en los que no se recomienda PA, tales como el cepillado o el uso de seda dental ${ }^{18,20,23,25,27,29,30,33,35}$. En la mayoría de los caso, estas bacteriemias no sobrepasan los $60 \mathrm{~min}$ de duración tras su generación $n^{17,25,26,29,32,34}$. Algunos estudios apoyan el uso de clorhexidina al 0,2\% previo a una intervención invasiva, para disminuir la carga bacteriana ${ }^{26,32,34}$.

\section{Discusión}

Los ensayos clínicos del ámbito odontológico, publicados después de las guías AHA y NICE (2007 y 2008 respectivamente), confluyen hacia una similitud cuantitativa y cualitativa de las bacteriemias producidas por diferentes procedimientos dentales, sean estos de carácter invasivo o no invasivo, inclusive aquellos causados por desempeños individuales de higiene buco-dentaria, lo que de alguna forma cuestiona, debido a su cotidianeidad y abasto, la necesidad de indicación de $\mathrm{PA}^{18,20,23,25,27,29,30,33,35}$.

Considerando la existencia de un consenso actual relacionado con la generación y caracterización de lo que conocemos como bacteriemias de origen odontológico ${ }^{18}$, y analizando el efecto que éste pudo generar en la evolución de las distintas guías de recomendación sanitarias publicadas en la última década -adicionado al gran aporte realizado por revisores especialistas en el área ${ }^{37-54}$ nos parece imperante comprender el impacto en ciertas regiones del mundo. En Inglaterra, hacia el año 2011, hubo una reducción de 78,6\% en las prescripciones de $\mathrm{PA}$-después de la introducción de la guía NICE- con aumento de incidencia en esta patología, pero no estableciéndose asociación causal por parte de los autores ${ }^{5,56}$. En Francia, la reducción de las indicaciones de PA no se tradujeron en una mayor incidencia de EI asociada al grupo viridans; pero los autores recomiendan una alerta respecto a Staphylococcus aureus ${ }^{1}$. En Estados Unidos por otro lado, coexisten conclusiones disímiles respecto a EI: algunos autores registran un aumento en la incidencia de ella ${ }^{57,58}$ y otros, no reportan cambios significativos ni aumentos perceptibles en la incidencia de EI asociada al grupo viridans ${ }^{59-60}$.

Sin embargo, actualmente los expertos subrayan como de alto valor preventivo una higiene bucal adecuada y control periódico con el odontólogo ${ }^{48}$. Es así como, la guía AHA (2015) "Endocarditis infecciosa en adultos: diagnóstico, terapia antimicrobiana y manejo de complicaciones", resume el abordaje en el ámbito estomatológico en tres puntos: a) pacientes con EI deben ser evaluados por un odontólogo para identificar y eliminar enfermedades bucales que predispongan a bacteriemia y puedan contribuir a EI recurrente; b) el examen clínico debe enfocarse en la inflamación de origen periodontal, la cual pudiere eventualmente, desencadenar infección pulpar y consecuentemente, absceso periodontal y c) resulta imperativo contar con exámenes radiográficos complementarios, del tipo retroalveolar total, para detectar elementos no visibles en la evaluación clínica ${ }^{4}$.

Los preceptos anteriores se establecieron contando con un nivel de evidencia tipo C (población evaluada muy limitada, principalmente basada en consenso de expertos) y de clase I (recomendación efectiva del procedimiento o tratamiento). El nivel de evidencia no ha cambiado significativamente desde la creación de la primera guía AHA en $1955^{10}$, reafirmando la necesidad de re-direccionar las investigaciones en esta área en una perspectiva que nos permita, junto a la evidencia actual, confirmar mediante mejores niveles de evidencia científica la influencia de los procedimientos dentales invasivos o cotidianos en el desarrollo de EI.

\section{Conclusión}

Las investigaciones publicadas en la última década, denotan una similitud en las bacteriemias producidas tras procedimientos dentales invasivos y no invasivos. Los autores destacan que un buen estado de salud periodontal, resultante de un control periódico con el odontólogo, es fundamental para la disminución en la exposición a bacteriemias. Procedimientos de higiene dental como el cepillado, en pacientes con enfermedad 
periodontal, podrían influir en el desarrollo de EI de un modo similar a lo constatado a los procedimientos dentales invasivos. Adicionalmente, la resistencia bacteriana es un problema actual reconocido y aceptado por la OMS ${ }^{61-65}$. Por ésta razón nos parece pertinente visibilizar la contradicción existente entre EI y administración -o no- de PA, tras bacteremias de origen dental, entendiendo que el fin último de éste artículo no es recomendar o instar hacia una postura sobre los puntos anteriormente expuestos, sino motivar una reflexión entorno a ellos.

En último caso, en el ámbito estomatológico, las decisiones por parte del cardiólogo serán las que primen ante las intervenciones que realizaremos a nuestros pacientes, aplicados al contexto individual establecido en cada país según sus Entidades de Salud y legislaciones propias.

Material suplementario en versión digital de la Revista Médica de Chile en: http://scielo.conicyt.cl

\section{Referencias}

1. Duval X, Delahaye F, Alla F, Tattevin P, Obadia JF, Le Moing V, et al; AEPEI Study Group. Temporal trends in infective endocarditis in the context of prophylaxis guideline modi - cations: three successive population-based surveys. J Am Coll Cardiol 2012; 59: 1968-76. doi: 10.1016/j.jacc.2012.02.029.

2. Correa de Sa DD, Tleyjeh IM, Anavekar NS, Schultz JC, Thomas JM, Lahr BD, et al. Epidemiological trends of infective endocarditis: a population-based study in Olmsted County, Minnesota [published correction appears in Mayo Clin Proc. 2010;85:772]. Mayo Clin Proc 2010; 85: 422-6.

3. Federspiel JJ, Stearns SC, Peppercorn AF, Chu VH, Fowler VG Jr. Increasing US rates of endocarditis with Staphylococcus aureus: 1999-2008. Arch Intern Med 2012; 172: 363-365. doi: 10.1001/ archinternmed.2011.1027.

4. Baddour LM, Wilson WR, Bayer AS, Fowler VG Jr, Tleyjeh IM, Rybak MJ, et al; on behalf of the American Heart Association Committee on Rheumatic Fever, Endocarditis, and Kawasaki Disease of the Council on Cardiovascular Disease in the Young, Council on Clinical Cardiology, Council on Cardiovascular Surgery and Anesthesia, and Stroke Council. Infective endocarditis in adults: diagnosis, antimicrobial therapy, and management of complications: a scientic statement for healthcare professionals from the American Heart As- sociation. Circulation 2015; 132: 00-00. DOI: 10.1161/ CIR.0000000000000296.

5. Oyonarte M, Montagna R, Braun S, Rojo P, Jara JL, Cereceda M, et al. Endocarditis infecciosa: características clínicas, complicaciones y mortalidad en 506 pacientes y factores pronósticos de sobrevida a 10 años (1998-2008). Estudio cooperativo nacional en endocarditis infecciosa en Chile (ECNEI-2). Rev Med Chile 2012; 140: 1517-28. ISSN 0034-9887. http://dx.doi.org/10.4067/S003498872012001200001.

6. Stockins B, Neira V, Paredes A, Castillo C, Troncoso A. Perfil clínico-epidemiológico de pacientes con endocarditis infecciosa, período 2003-2010 en el hospital de Temuco, Chile. Rev Med Chile 2012; 140: 1304-1311. http://dx.doi.org/10.4067/S0034-98872012001000010.

7. Flores P, González N, Betancourt P, Berho J, Astudillo C, García C, et al. Endocarditis Infecciosa: caracterización clínica de la enfermedad. Revisión de casos de los últimos 5 años. Rev Chil Cardiol 2017; 36: 34-40. http:// dx.doi.org/10.4067/S0718-85602017000100004.

8. Osler W. Gulstonian lectures on malignant endocarditis. lecture I, and lecture II. Lancet 1885; 1: 415- 8, 459- 64.

9. Okell CC, Elliott SD. Bacteremia and oral sepsis: with special reference to the aetiology of subacute endocarditis. Lancet 1935; 2: 869-72.

10. Jones TD, Baumgartner L, Bellows MT, Breese BB, Kuttner AG, McCarty M, et al (Committee on Prevention of Rheumatic Fever and Bacterial Endocarditis, American Heart Association). Prevention of rheumatic fever and bacterial endocarditis through control of streptococcal infections. Circulation 1955; 11: 317-20.

11. Wilson W, Taubert KA, Gewitz M, et al. AHA Guideline: Prevention of Infective Endocarditis: Guidelines From the American Heart Association: A Guideline From the American Heart Association Rheumatic Fever, Endocarditis, and Kawasaki Disease Committee, Council on Cardiovascular Disease in the Young, and the Council on Clinical Cardiology, Council on Cardiovascular Surgery and Anesthesia, and the Quality of Care and Outcomes. Circulation 2007; 116: 1736-54.

12. 2015 ESC Guidelines for the management of Infective endocarditis. European Society of Cardiology. European Heart Journal 2015; 36: 3075-123.

13. Nishimura RA, Otto CM, Bonow RO, Carabello BA, Erwin JP 3rd, Fleisher LA, et al. 2017 AHA/ACC focused update of the 2014 AHA/ACC guideline for the management of patients with valvular heart disease: a report of the American College of Cardiology/American Heart Association Task Force on Clinical Practice Guidelines. National Institute for Health and Care Excellence London Prophylaxis Against Infective Endocarditis: 
Antimicrobial Prophylaxis Against Infective Endocarditis in Adults and Children Undergoing Interventional Procedures. CG64: 2008. J Am Coll Cardiol 2017; 70: 252-89.

14. National Institute for Health and Care Excellence London. Prophylaxis against infective endocarditis. Antimicrobial prophylaxis against infective endocarditis in adults and children undergoing interventional procedures. March 2008. Last updated: July 2016.

15. Antibiotics for the prophylaxis of bacterial endocarditis in dentistry. Cochrane Database of Syst Rev 2013; 10: Art. No: CD003813. DOI:10.1002/14651858.CD003813. pub4.

16. Tomás I, Álvarez M, Limeres J, Potel C, Medina J, Diz $\mathrm{P}$. Prevalence, duration and aetiology of bacteraemia following dental extractions. Oral Diseases 2007; 13: 56-62. doi:10.1111/j.1601-0825.2006.01247.x

17. Lockhart PB, Brennan MT, Sasser HC, Fox PC, Paster BJ, Bahrani-Mougeot FK. Bacteremia associated with toothbrushing and dental extraction. Circulation 2008; 117 (24): 3118-25. doi:10.1161/CIRCULATIONAHA.107.758524.

18. Maestre Vera JR, Mateo Maestre M, Sánchez Santana P. Bacteriemia secundaria a procedimientos odontológicos periodontales. Rev Esp Quimioter 2008; 21 (3): 153-6.

19. Crasta K, Daly CG, Mitchell D, Curtis B, Stewart D, Heitz-Mayfield LJ. Bacteraemia due to dental flossing. J Clin Periodontol 2009; 36: 323-32.

20. Gürel HG, Basciftci FA, Arslan U. Transient bacteremia after removal of a bonded maxillary expansion appliance. Am J Orthod Dentofacial Orthop 2009; 135: 190-3.

21. Babu D, Reddy N, Swaroop D, Babu K, Kiran, Swaminathan M. Evaluation Of Bacteremia Following Periodontal Probing In Gingivitis And Periodontitis Patients. The Internet Journal of Dental Science. 2009 Volume 9 Number 2.

22. Hussein EA, Acar A, Dogan AA, Kadir T, Caldemir S, Erverdi N. Investigation of bacteremia after toothbrushing in orthodontic patients. Korean J Orthod 2009; 39 (3): $177-84$.

23. Nixon PP, Littler P, Davies K, Krishnam MS. Does sialography require antibiotic prophylaxis? Br J Radiol. 2009; 82 (981): 732-4. doi: 10.1259/bjr/23033283.

24. Lockhart PB, Brennan MT, Thornhill M, Michalowicz BS, Noll J, Bahrani-Mougeot FK, et al. Poor oral hygiene as a risk factor for infective endocarditis-related bacteremia. J Am Dent Assoc 2009; 140: 1238-44.

25. Piñeiro A, Tomás I, Blanco J, Álvarez M, Seoane J, Diz P. Bacteraemia following dental implants' placement. Clin. Oral Impl Res 2010; 21: 913-8. doi: 10.1111/j.16000501.2010.01928.x.
26. Dubey R, Jalili VP, Jain S, Dubey A. Transient bacteremia consequent to tooth brushing in orthodontic patients. Prog Orthod 2012; 13 (3): 237-45. doi: 10.1016/j. pio.2012.02.004.

27. Bölükbas 1 N, Özdemir T, Öksüz L, Gürler N. Bacteremia following dental implant surgery: Preliminary results. Med Oral Patol Oral Cir Bucal 2012; 17 (1): e69-75.

28. Chen SJ, Liu CJ, Chao TF, Wang KL, Wang FD, Chen $\mathrm{TJ}$, et al. Dental scaling and risk reduction in infective endocarditis: a nationwide population-based case-control study. Can J Cardiol 2013; 29 (4): 429-33. doi: 10.1016/j.cjca.2012.04.018.

29. Zhang W, Daly CG, Mitchell D, Curtis B. Incidence and magnitude of bacteraemia caused by flossing and by scaling and root planing. J Clin Periodontol 2013; 40 (1): 41-52. doi: 10.1111/jcpe.12029.

30. Ileri Z, Akin M, Aybuke Erdur E, Turk Dagi H, Findik D. Bacteremia after piezocision. American Journal of Orthodontics and Dentofacial Orthopedics October 2014 Vol 146 Issue 4.

31. Ugwumba CU, Adeyemo WL, Odeniyi OM, Arotiba GT, Ogunsola FT. Preoperative administration of $0.2 \%$ chlorhexidine mouthrinse reduces the risk of bacteraemia associated with intra-alveolar tooth. J Craniomaxillofac Surg 2014; 42: 783-1788. http://dx.doi. org/10.1016/j.jcms.2014.06.015.

32. Mougeot FK, Saunders SE, Brennan MT, Lockhart $\mathrm{PB}$. Associations between bacteremia from oral sources and distant-site infections: tooth brushing versus single tooth extraction. Oral Surg Oral Med Oral Pathol Oral Radiol 2015; 119 (4): 430-5. doi: 10.1016/j. oooo.2015.01.009.

33. Barbosa M, Prada-López I, Álvarez M, Amaral B, de los Angeles Casares-De-Cal M, Tomás I. Post-Tooth Extraction Bacteraemia: A Randomized Clinical Trial on the Efficacy of Chlorhexidine Prophylaxis. PLoS ONE 201510 (5): e0124249. doi:10.1371/journal. pone.0124249.

34. Chirillo F, Faggiano P, Cecconi M, Moreo A, Squeri A, Gaddi O, et al. Predisposing cardiac conditions, interventional procedures, and antibiotic prophylaxis among patients with infective endocarditis. Italian Registry on Infective Endocarditis (RIEI) Investigators. Am Heart J. 2016; 179: 42-50. doi: 10.1016/j.ahj.2016.03.028.

35. Tubiana S, Blotière PO, Hoen B, Lesclous P, Millot $\mathrm{S}$, Rudant J, et al. Dental procedures, antibiotic prophylaxis, and endocarditis among people with prosthetic heart valves: nationwide population based cohort and a case crossover study. BMJ 2017; 358: j3776. doi: 10.1136/bmj.j3776. 
36. Carmona I, Diz Dios P, Scully C. Efficacy of antibiotic prophylactic regimens for the prevention of bacterial endocarditis of oral origin. J Dent Res 2007; 86 (12): 1142-59.

37. Daly CG, Currie BJ, Jeyasingham MS, Moulds RF, Smith JA, Strathmore NF, et al. A change of heart: the new infective endocarditis prophylaxis guidelines. Aust Dent J 2008; 53 (3): 196-200; quiz 297. doi: 10.1111/j.18347819.2008.00049.x.

38. Lam DK, Jan A, Sándor GK, Clokie CM; American Heart Association. Prevention of infective endocarditis: revised guidelines from the American Heart Association and the implications for dentists. J Can Dent Assoc 2008; 74 (5): 449-53.

39. Poveda-Roda R, Jiménez Y, Carbonell E, Gavaldá C, Margaix-Muñoz MM, Sarrión-Pérez G. Bacteremia originating in the oral cavity. A review. Med Oral Patol Oral Cir Bucal 2008; 13 (6): E355-62.

40. Seymour RA, Hogg SD. Antibiotics and chemoprophylaxis. Periodontol 2000. 2008; 46: 80-108. doi: 10.1111/j.1600-0757.2008.00246.x.

41. Shanson D. New British and American guidelines for the antibiotic prophylaxis of infective endocarditis: do the changes make sense? A critical review. Curr Opin Infect Dis 2008; 21 (2): 191-9. doi: 10.1097/QCO. 0b013e3282f94765.

42. Wright TI, Baddour LM, Berbari EF, Roenigk RK, Phillips PK, Jacobs MA, et al. Antibiotic prophylaxis in dermatologic surgery: advisory statement 2008. J Am Acad Dermatol 2008; 59 (3): 464-73. doi: 10.1016/j. jaad.2008.04.031.

43. Bascones-Martínez A, Muñoz-Corcuera M, Meurman $\mathrm{JH}$. Odontogenic infections in the etiology of infective endocarditis. Cardiovasc Hematol Disord Drug Targets 2009; 9 (4): 231-5.

44. Brooks N. Prophylactic antibiotic treatment to prevent infective endocarditis: new guidance from the National Institute for Health and Clinical Excellence. Heart 2009; 95 (9): 774-80. doi: 10.1136/hrt.2008.147702.

45. Gopalakrishnan PP, Shukla SK, Tak T. Infective endocarditis: rationale for revised guidelines for antibiotic prophylaxis. Clin Med Res 2009; 7 (3): 63-8. doi: 10.3121/cmr.2009.848.

46. FitzGerald K, Fleming P, Franklin O. Dental health and management for children with congenital heart disease. Prim Dent Care 2010; 17 (1): 21-5. doi: 10.1308/135576110790307690.

47. Persac S, Prévost R, Hardy H, Gigon S, Peron JM.[An update on focal infection of oral origin]. Rev Stomatol Chir Maxillofac 2011; 112 (6): 353-9. doi: 10.1016/j. stomax.2011.10.004.
48. Tomás I, Diz P, Tobías A, Scully C, Donos N. Periodontal health status and bacteraemia from daily oral activities: systematic review/meta-analysis. J Clin Periodontol 2012; 39: 213-28.

49. Bascones-Martínez A, Muñoz-Corcuera M, Bascones-Ilundain J. Relationship between odontogenic infections and infective endocarditis. Med Clin (Barc) 2012; 138 (7): 312-7. doi: 10.1016/j.medcli.2011.03.029.

50. Weiss A, Dym H. Review of antibiotics and indications for prophylaxis. Dent Clin North Am 2012; 56 (1): 23544, x. doi: 10.1016/j.cden.2011.07.003.

51. Costantinides F, Clozza E, Ottaviani G, Gobbo M, Tirelli G, Biasotto M. Antibiotic prophylaxis of infective endocarditis in dentistry: clinical approach and controversies. Oral Health Prev Dent 2014; 12 (4): 305-11. doi: 10.3290/j.ohpd.a32133.

52. Mang-de la Rosa MR, Castellanos-Cosano L, Romero-Perez MJ, Cutando A. The bacteremia of dental origin and its implications in the appearance of bacterial endocarditis. Med Oral Patol Oral Cir Bucal 2014; 19 (1): e67-74.

53. Hossaini-zadeh M. Current Concepts of Prophylactic Antibiotics for Dental Patients. Dent Clin North Am 2016; 60 (2): 473-82. doi: 10.1016/j.cden.2015.12.002.

54. Thornhill MH, Dayer MJ, Forde JM, Corey GR, Chu $\mathrm{VH}$, Couper DJ, et al. Impact of the NICE guideline recommending cessation of antibiotic prophylaxis for prevention of infective endocarditis: before and after study. BMJ 2011; 342: d2392. doi: 10.1136/bmj.d2392.

55. Dayer MJ, Jones S, Prendergast B, Baddour LM, Lockhart $\mathrm{PB}$, Thornhill MH. Incidence of infective endocarditis in England, 2000-2013: a secular trend, interrupted time-series analysis. Lancet 2015; 385: 1219-28.

56. Bor DH, Woolhandler S, Nardin R, Brusch J, Himmelstein DU. Infective Endocarditis in the U.S., 1998-2009: A Nationwide Study. PLoS ONE 2013; 8 (3): e60033. doi:10.1371/journal.pone.0060033.

57. Pant S, Patel NJ, Deshmukh A, Golwala H, Patel N, Badheka A, et al. Trends in Infective Endocarditis Incidence, Microbiology, and Valve Replacement in the United States From 2000 to 2011. J Am Coll Cardiol 2015; 65: 2070-6.

58. Desimone DC, Tleyjeh IM, Correa de Sa DD, Anavekar NS, Lahr BD, Sohail MR, et al. Incidence of infective endocarditis caused by viridans group streptococci before and after publication of the 2007 American Heart Association's endocarditis prevention guidelines. Circulation 2012; 126: 60-4.

59. Pasquali SK, He X, Mohamad Z, McCrindle BW, Newburger JW, Li JS, et al. Trends in endocarditis hospita- 
lizations at US children's hospitals: impact of the 2007 American Heart Association Antibiotic Prophylaxis Guidelines. Am Heart J 2012; 163: 894-99.

60. Bikdeli B, Wang Y, Kim N, Desai MM, Quagliarello $\mathrm{V}$, Krumholz HM. Trends in hospitalization rates and outcomes of endocarditis among Medicare beneficiaries. J Am Coll Cardiol 2013; 62: 2217-26.

61. Sistema mundial de vigilancia de la resistencia a los antimicrobianos: manual para la primera fase de implementación [Global antimicrobial resistance surveillance system: manual for early implementation]. Ginebra: Organización Mundial de la Salud; 2017. Licencia: CC BY- NC-SA 3.0 IGO.

62. Liu YY, Wang Y, Walsh TR, Yi LX, Zhang R, Spencer J, et al. Emergence of plasmid-mediated colistin resistance mechanism mcr-1 in animals and human beings in China: a microbiological and molecular biological study.
Lancet Infect Dis 2016; 16: 161-8.

63. McGann P, Snesrud E, Maybank R, Corey B, Ong AC, Clifford R, et al. Escherichia coli harboring mcr-1 and blaCTX-M on a novel IncF plasmid: first report of mcr1 in the United States. Antimicrob Agents Chemother 2016; 60: 4420-1.

64. Castanheira M, Griffin MA, Deshpande LM, Mendes RE, Jones RN, Flamm RK. Detection of mcr-1 among Escherichia coli clinical isolates collected worldwide as part of the SENTRY Antimicrobial Surveillance Program during 2014 and 2015. Antimicrob Agents Chemother 2016; 60: 5623-4.

65. Xavier BB, Lammens C, Ruhal R, Kumar-Singh S, Goossens $\mathrm{H}$, Malhotra-Kumar S. Identification of a novel plasmid-mediated colistin- resistance gene, mcr-2, in Escherichia coli, Belgium, June 2016. Euro Surveill 2016; 21. 